(c) Auteures. Cette œuvre, disponible à http://dx.doi.org/10.18162/fp.2020.519, est distribuée sous licence Creative Commons Attribution 4.0 International http://creativecommons.org/licences/by/4.0/deed.fr
Marie-Ève Gadbois Université du Québec à Montréal (Canada) France Dubé Université du Québec à Montréal (Canada)

\section{Adaptation d'un protocole de collaboration basé sur les niveaux d'interdépendance et les besoins des intervenants au sein d'une école primaire soutenant la scolarisation d'élèves ayant un TSA en classe ordinaire}

\author{
Adaptation of a collaboration protocol based on levels of \\ interdependence and school actors'needs in a primary school \\ supporting mainstreaming of students with ASD in regular
}

Cet article présente une recherche-développement proposant l'adaptation d'un protocole visant à soutenir la collaboration entre les intervenants scolaires impliqués lors de l'intégration d'élèves ayant un trouble du spectre de l'autisme (TSA). Les objectifs de cette recherche étaient : 1) d'étudier les pratiques

collaboratives selon le modèle théorique du continuum d'interdépendance de Beaumont, Lavoie et Couture (2010) et 2) d'adapter un protocole déjà existant pour soutenir la collaboration et mieux répondre aux besoins du milieu. Les pratiques collaboratives étudiées atteignaient des niveaux intermédiaires d'interdépendance. Des adaptations ont été proposées en vue d'améliorer le protocole déjà en place : la centralisation des informations partagée et la planification de rencontres en cours d'année scolaire ont été ajoutées au protocole.

\section{Mots-clés}

Intégration scolaire, pratiques collaboratives, élève ayant un trouble du spectre de l'autisme (TSA), organisation scolaire, recherche-développement.

Abstract

This article presents a development research proposing a protocol aiming at supporting the collaboration between school actors involved in the mainstreaming of a student with autism spectrum disorder (ASD). The objectives of this study were to 1) study collaborative practices based on the theoretical model of the interdependence continuum (Beaumont et al., 2010) and 2) adapt a protocol to support collaboration and better meet the needs of the school actors. The results showed that collaborative practices in this school reached intermediate levels of interdependence. Adaptations have been proposed to the protocol already in place: organized monitoring between the actors involved and effective planned meetings during the school year among other things, have been added to the protocol.

Keywords

Mainstreaming, collaborative practices, students with autism spectrum disorder (ASD), school organization, development research.
Au Québec, depuis les années soixante, de nombreuses actions ont été mises en place pour soutenir les élèves ayant des besoins particuliers dans les milieux scolaires. Depuis la publication du rapport du Comité provincial de l'enfance inadaptée (COPEX), en 1976, l'intégration des élèves ayant des besoins particuliers dans l'école et dans les classes ordinaires est une pratique privilégiée (Boutin et Bessette, 2009; Goupil, 2014). Depuis la parution de ce rapport, de nombreux textes de lois et des prescriptions ministérielles ont été publiés, notamment par le ministère de l'Éducation, du Loisir et du Sport (MELS, 2007; 2010), en vue de favoriser des pratiques adaptées à chaque élève en fonction de ses besoins et capacités. Parmi ces encadrements figure la Politique de l'adaptation scolaire qui stipule que, si l'évaluation individuelle des besoins et capacités de l'élève révèle que l'intégration, partielle ou complète, en classe ordinaire est la mesure la plus profitable pour maximiser les apprentissages et l'insertion sociale de l'élève, les services éducatifs doivent être organisés afin de permettre cette intégration (Ministère de l'Éducation du Québec [MEQ], 1999).

Considérant cette Politique, une commission scolaire de la grande région de Montréal a orienté ses modalités d'organisation des services éducatifs en privilégiant l'intégration en classe ordinaire pour les élèves ayant des besoins particuliers. Selon les textes d'encadrement de cette commission scolaire, les modalités d'organisation des services éducatifs doivent être de nature à faciliter les apprentissages et l'insertion sociale de l'élève et doivent favoriser l'ouverture à la différence. Au sein de la commission scolaire, des comités consultatifs concernant les élèves ayant des besoins particuliers ont vu le jour et des démarches concernant l'intégration d'élèves vers la classe ordinaire se sont organisées. C'est à travers un de ces comités consultatifs composés d'une équipe d'enseignants et de conseillers 
pédagogiques qu'a été conçu un protocole d'intégration partielle intitulé Protocole d'intégration partielle en cours d'année pour un élève en classe spéciale vers la classe ordinaire. Ce protocole décrit et encadre une démarche, à l'intention des intervenants scolaires, pouvant être mise en place en cours d'année scolaire afin de soutenir la collaboration dans le cadre de l'intégration d'élèves ayant des besoins particuliers vers la classe ordinaire.

Dans sa plus simple définition, l'intégration partielle signifie: «le processus par lequel un élève participe pour une partie de son temps de présence à l'école à des activités d'apprentissage d'un groupe d'élèves handicapés ou en difficulté d'adaptation ou d'apprentissage et est pour l'autre partie de son temps intégré dans un groupe ordinaire » (MELS, 2010, p. 10). Dans un contexte où l'intégration est souhaitable pour les élèves, les milieux scolaires planifient, en cours d'année scolaire, de faire transiter les élèves scolarisés en classe spécialisée vers la classe ordinaire, pour certaines périodes précises : l'élève chemine alors entre deux classes et la collaboration entre les intervenants impliqués au sein de cette intégration s'avère d'une grande importance.

\section{Contexte spécifique de la recherche}

Cette recherche s'est tenue dans une école primaire régulière dans laquelle se retrouvent trois classes spécialisées pour élèves ayant un trouble du spectre de l'autisme (TSA). Au moment de la répartition des élèves dans ces trois classes spécialisées, l'équipe-école a fait le choix de grouper les élèves en fonction de leur niveau d'autonomie et de leur capacité, selon eux, à être intégrés en classe ordinaire en cours d'année scolaire. Ainsi, huit élèves ont été rassemblés dans un groupe-classe. Cinq d'intervenantes ${ }^{1}$ impliquées dans la scolarisation d'un élève ayant un TSA scolarisé dans une classe spécialisée et intégré à temps partiel dans une classe ordinaire de $5^{\mathrm{e}}$ année du primaire ont pris part à la recherche. Au moment de la recherche, cet élève était donc scolarisé dans une classe spécialisée, mais participait aux activités éducatives, pour quelques périodes ciblées par semaine, dans la classe ordinaire, soutenu, au besoin, par l'éducatrice spécialisée. Il importe de mentionner que, selon les intervenantes, l'intégration partielle était la mesure la plus souhaitable à ce moment pour l'élève, mais que l'équipe-école souhaitait proposer progressivement une scolarisation à temps plein, en classe ordinaire, pour cet élève. En cours d'année scolaire, les intervenantes ont constaté que certaines modalités relatives à la collaboration en vue de soutenir l'intégration de l'élève n'avaient pas clairement été définies. Les intervenantes n'avaient pas de moment réservé ni de procédure favorisant la collaboration et une question était évoquée sans cesse : à qui la responsabilité concernant l'enseignement, l'évaluation et les interventions auprès de cet élève revient-elle? On a donc constaté que les responsabilités attendues de la part de chaque intervenante et les modalités de collaboration devaient être précisées.

\section{Objectifgénéral}

Dans le cadre de la présente recherche, les intervenantes ont été interrogées au sujet des modalités liées au protocole d'intégration préalablement élaboré dans cette commission scolaire pour connaître le niveau d'utilisation de ce protocole et son appréciation. Même si ce protocole existait depuis quelques années, il devait être analysé afin de valoriser son potentiel en vue de proposer une démarche pour rehausser le niveau de collaboration lors de l'intégration vers la classe ordinaire d'un élève ayant un TSA. 
Adaptation d'un protocole de collaboration basé sur les niveaux d'interdépendance et les besoins des intervenants au sein d'une école primaire soutenant la scolarisation d'élèves ayant un TSA en classe ordinaire

Dans une perspective de réussite pour tous les élèves à travers laquelle il serait souhaitable d'assurer la collaboration entre les intervenants œuvrant en milieu scolaire, l'objectif général de la recherche était d'adapter le protocole d'intégration en vue de soutenir une démarche pour rehausser la collaboration entre les intervenants lors de l'intégration d'un élève ayant un TSA vers la classe ordinaire.

Les niveaux d'interdépendance comme modèle théorique abordant la collaboration en milieu scolaire Les niveaux de collaboration en place entre les cinq intervenantes participantes au moment de la recherche ont été analysés à l'aide du modèle de Little (1990). Ce modèle théorique propose un continuum basé sur quatre niveaux d'interdépendance au sein de la collaboration dans les milieux scolaires. Les pratiques collaboratives, qui s'étalent sur un continuum, passent de l'autonomie professionnelle de chaque intervenant jusqu'à l'établissement d'un travail conjoint entre ceux-ci. Ce modèle théorique a été opérationnalisé par Beaumont et al. (2010) pour permettre de situer les pratiques collaboratives en milieux scolaires. Pour faciliter l'analyse des pratiques collaboratives au sein des milieux scolaires, nous avons adapté le modèle proposé par Beaumont et al. (2010) (tableau 1).

\section{Tableau 1}

Les niveaux d'interdépendance (Gadbois, 2015), adapté de Beaumont et al. (2010)

\begin{tabular}{|c|c|c|c|}
\hline \multicolumn{2}{|c|}{ Indépendance } & \multicolumn{2}{|c|}{ Interdépendance } \\
\hline Niveau 1 & Niveau 2 & Niveau 3 & Niveau 4 \\
\hline Échanges de nature sociale & Relation d'entraide & $\begin{array}{l}\text { Discussions pédagogiques } \\
\text { ou disciplinaires }\end{array}$ & $\begin{array}{l}\text { Partage et chevauchement } \\
\text { d'expertise }\end{array}$ \\
\hline $\begin{array}{l}\text { - Échanges informels } \\
\text { - Échanges superficiels } \\
\text { - Échanges anecdotiques }\end{array}$ & $\begin{array}{l}\text { - Relation de type informel } \\
\text { - Relation de type } \\
\text { ponctuelle } \\
\text { - Offres et demandes d'aide } \\
\text { - À sens unique }\end{array}$ & $\begin{array}{l}\text { - Partage d'idées } \\
\text { - Partage de matériel } \\
\text { - Planification de } \\
\text { stratégies d'enseignement }\end{array}$ & $\begin{array}{l}\text { - Cadre commun de référence } \\
\text { - Débats d'opinion } \\
\text { - Partage d'expertise, } \\
\text { de ressources, et de } \\
\text { responsabilité } \\
\text { - Résolutions de problèmes } \\
\text { complexes }\end{array}$ \\
\hline
\end{tabular}

Tels que présentés sur le tableau 1, les niveaux de collaboration s'opérationnalisent sous quatre niveaux d'interdépendance en fonction de la fréquence et de l'intensité des interactions ainsi que des possibilités d'influence mutuelle entre les intervenants. Le niveau 1 implique des échanges anecdotiques et de nature sociale entre les intervenants. Au niveau 2, une relation d'entraide s'installe entre ceux-ci et un soutien est offert à la demande de l'autre. À ce niveau, les échanges se font de façon informelle et ponctuelle. Ensuite, le niveau 3 implique des discussions planifiées, des échanges réciproques ainsi qu'un partage de stratégies pédagogiques ou de matériel à mettre en place. Finalement, le niveau 4, le partage d'expertise entre les intervenants, implique la planification à long terme d'objectifs communs et la résolution de problèmes complexes.

Afin de proposer une démarche qui contribue à soutenir la collaboration entre les intervenantes au cours d'une intégration vers la classe ordinaire pour un élève ayant un TSA, cette recherche s'est penchée sur les pratiques collaboratives entre cinq intervenantes scolaires ainsi que sur leurs besoins concernant les adaptations à mettre en place en vue de rehausser cette collaboration. Plus précisément, les objectifs poursuivis par cette recherche étaient: 1) d'étudier les pratiques collaboratives lors de 
l'intégration vers la classe ordinaire d'un élève ayant un TSA en s'appuyant sur le modèle théorique du continuum d'interdépendance de Beaumont et al. (2010) et 2) d'adapter un protocole pour soutenir la collaboration au moment de cette intégration et pour répondre aux besoins du milieu. Cette recherche reflète la volonté de soutenir la réflexion et de proposer un protocole d'intégration en vue de soutenir une démarche pour rehausser la collaboration au cours de l'intégration d'élèves ayant un TSA en classe ordinaire.

\section{Méthodologie}

Cette recherche-développement à devis qualitatif s'est inscrite dans une démarche de compréhension et d'interprétation impliquant les acteurs concernés dans leur environnement social spécifique (Boutin, 1997). Ce type de recherche inclut le développement de matériel pédagogique, mais aussi le développement de stratégies ou de méthodes ayant des incidences sur l'action éducative (Loiselle et Harvey, 2007). L'enjeu de cette recherche-développement se voulait pragmatique; le but étant orienté vers une solution fonctionnelle qui se veut correctrice d'une problématique vécue dans le milieu. Par l'analyse des besoins du milieu scolaire qui a été étudié, un protocole impliquant une démarche de collaboration entre les intervenants scolaires dans le cadre de l'intégration vers la classe ordinaire d'un élève ayant un TSA a été adapté à partir de celui déjà en place et a ensuite été proposé aux intervenantes participantes (Van der Maren, 2003).

\section{Participantes}

Une enseignante en classe ordinaire de $5^{\mathrm{e}}$ année du primaire, une technicienne en éducation spécialisée (TES), une enseignante en classe spécialisée pour élèves ayant un TSA et la directrice d'école sont les participantes qui ont pris part à la recherche. Au sein du processus d'intégration étudié pour cette recherche, l'enseignante de la classe spécialisée est celle qui est titulaire de l'élève intégré et l'enseignante de la classe ordinaire est celle qui accueille l'élève dans sa classe lors des périodes d'intégration. La technicienne en éducation spécialisée est présente auprès de l'élève dans les moments où il a besoin de soutien : elle est présente auprès de lui, peu importe la classe dans laquelle l'élève se retrouve. Ainsi, la TES peut être présente avec l'élève en classe ordinaire lors des périodes d'intégration, si sa présence est nécessaire à la tâche à accomplir par l'élève. Les rencontres avec ces intervenantes nous ont fait comprendre la place significative que prenait la conseillère pédagogique lors de l'intégration vers la classe ordinaire d'élèves ayant un TSA. Ainsi, au cours de la recherche-développement, la conseillère pédagogique en adaptation scolaire a également pris part au projet. Notre recherche implique donc ces cinq intervenantes qui collaborent pour soutenir la scolarisation d'un élève ayant un TSA qui avait, au départ, été intégré à temps partiel en vue d'être progressivement intégré totalement dans une classe ordinaire.

\section{Entretiens semi-dirigés}

Afin d'étudier les pratiques collaboratives mises en place dans le cadre de l'intégration vers la classe ordinaire en vue d'adapter le protocole déjà existant, nous avons mené des entretiens semi-dirigés avec les participantes. Ce type d'entretien a permis de recueillir des données centrées sur les participantes 
du projet et sur la perception qu'elles ont au sujet des thèmes abordés (Boutin, 2011). Ces entretiens comportaient trois sections : 1) les informations relatives à chaque intervenante (qualification, années d'expérience, etc.); 2) les perceptions de la collaboration vécue entre les différentes intervenantes dans le cadre d'intégration vers la classe ordinaire; 3) les besoins des intervenantes face à la collaboration vécue pour soutenir cette intégration. Les entretiens étaient d'une durée variant de 30 à 65 minutes et, au besoin, certaines intervenantes ont complété leurs propos en faisant parvenir des informations complémentaires par courriel à la chercheure.

\section{Analyses}

Les verbatim d'entretiens concernant les deux premières sections ont été analysés en se basant sur un codage fermé selon le modèle théorique des quatre niveaux d'interdépendance proposés par Beaumont et al. (2010) (tableau 1). Les propos recueillis ont été classés dans une matrice (Miles et Huberman, 1994) afin de faire ressortir les pratiques collaboratives énoncées pour chaque niveau d'interdépendance. En contrepartie, les pratiques mentionnées lors des entretiens qui étaient situées hors des niveaux d'interdépendance ont également été regroupées dans une matrice. Les données de la troisième section de l'entretien concernant les besoins des intervenantes ont quant à elles été analysées à partir d'un codage ouvert basé sur les thèmes émergents. Dans cette section d'entretien, les besoins des intervenants et les obstacles à la collaboration ont été abordés en plus de pistes de solution. Ces données ont été analysées afin d'apporter au protocole les adaptations nécessaires pour contribuer à atteindre un plus haut niveau de collaboration.

Les adaptations proposées pour le Protocole d'intégration partielle en cours d'année pour un élève en classe spéciale vers la classe ordinaire s'appuient sur le modèle théorique de Beaumont et al. (2010) ainsi que sur les besoins des intervenantes. Ces adaptations visent à permettre l'atteinte de pratiques collaboratives relevant d'un plus haut niveau d'interdépendance (Beaumont et al., 2010).

\section{Résultats}

\section{Pratiques collaboratives lors de l'intégration vers la classe ordinaire d'un élève ayant un TSA}

Les résultats présentent la collaboration, telle que perçue par les intervenantes participantes concernant l'intégration vers la classe ordinaire pour un élève ayant un TSA. Lors des entretiens, les intervenantes ont rapporté peu d'exemples de pratiques collaboratives relevant du niveau 1 de Beaumont et al. (2010) concernant les échanges de nature sociale. Les propos recueillis concernant les modalités des rencontres nous ont confirmé que celles-ci sont planifiées de façon spontanée et se tiennent de manière informelle. Les sujets les plus souvent abordés lors des discussions sont l'organisation de l'horaire et les situations problématiques survenues. Certains aspects de la collaboration relèvent du niveau 2 d'interdépendance : les rencontres surviennent surtout à la demande d'une intervenante envers une autre et elles sont majoritairement vécues entre deux intervenantes (peu de rencontres planifiées à plus de deux intervenantes). Selon la directrice, des rencontres plus formelles se tiennent lorsque des problèmes spécifiques surviennent: "Quand il y a des problématiques particulières, on prend du temps pour se rencontrer, en équipe-multi. Quand ce sont des problèmes au quotidien, ce sont les [enseignants] entre eux, qui se donnent du temps [Directrice].» 
En ce qui a trait aux discussions pédagogiques ou disciplinaires (niveau 3), les échanges portent majoritairement sur les stratégies d'intervention pour soutenir l'élève ayant un TSA. À travers les échanges entre les intervenantes, on y constate peu de partage concernant la planification de stratégies communes d'enseignement ou encore de partage de matériel. En début d'année scolaire, une organisation pour contribuer à établir une collaboration étroite entre les deux enseignantes avait été mise en place. Des rencontres avaient été planifiées de façon hebdomadaire afin de préparer la semaine à venir et de cibler les périodes d'intégration. Or, ces rencontres se sont tenues seulement sur une période de deux semaines et sont devenues plus rares par la suite pour des raisons variées : problèmes ponctuels ou imprévus en classe. Les intervenantes ont expliqué que leurs grilles horaires respectives n'étaient pas planifiées pour leur permettre de se rencontrer; elles n'avaient pas de périodes libres communes, ce qui rendait la planification de rencontres complexe.

Finalement, concernant le partage d'expertise attendu au niveau 4 d'interdépendance, les participantes ont évoqué que les rôles au moment de la planification de l'intégration étaient vaguement définis. Certaines décisions basées sur des buts communs ont été évoquées et concernaient exclusivement l'utilisation du plan d'intervention adapté (PIA) comme point d'ancrage aux interventions mises en place dans le cadre de l'intégration de lélève vers la classe ordinaire. En effet, les objectifs ciblés dans le PIA étaient connus par toutes les intervenantes et les actions étaient mises en place pour atteindre ces objectifs. Relativement à la résolution de problèmes complexes et à la planification de l'intégration, les intervenantes nous ont mentionné que les décisions étaient plutôt prises à court terme et qu'elles étaient peu planifiées dans le temps.

\section{Obstacles aux pratiques collaboratives}

De nombreux obstacles à la collaboration lors de l'intégration vers la classe ordinaire ont été soulevés lors des entretiens avec les participantes. Tout d'abord, le manque de temps pour se rencontrer a été nommé à plusieurs reprises. À cet effet, l'enseignante de la classe spécialisée et l'éducatrice spécialisée expliquent : "C'est un élève qui aurait eu besoin qu'on puisse s'asseoir, mais on n'a pas le temps [Enseignante de la classe spécialisée]. » "On essaie de se voir une fois par jour, le matin, mais ça dépend si on a du temps [Éducatrice spécialisée].»

Par ailleurs, les intervenantes évoquent un certain manque de communication entre les diverses intervenantes et les informations concernant l'élève et son intégration ne circulent pas toujours entre chacune d'entre elles :

C'est un élève qui demande une très grande cohérence, avec la présence des trois intervenantes, c'est un élève qui se faufle facilement dès qu'il voit une zone grise. Alors il faut vraiment être cohérent; si on dit on essaie quelque chose, on doit vraiment le faire toutes les trois [les deux enseignantes et la TES] parce que sinon, il se faufile. [Enseignante de la classe spécialisée]

Une autre problématique soulevée concerne les rôles et responsabilités de chaque intervenante. En effet, selon elles, les rôles semblent vaguement définis en ce qui concerne l'intégration vers la classe ordinaire de l'élève concerné : 
Adaptation d'un protocole de collaboration basé sur les niveaux d'interdépendance et les besoins des intervenants au sein d'une école primaire soutenant la scolarisation d'élèves ayant un TSA en classe ordinaire

Un autre défi : le rôle des enseignants. Ils sont chacun dans leur classe, mais quiest-ce qui est de la responsabilité de l'une et l'autre? [...] Où commence leur responsabilité? Où s'arrête-t-elle? Ca, c'est une zone floue qui nécessite [d'être abordée]. [Directrice].

Finalement, les participantes ont aussi fait mention des changements de personnel scolaire en cours d'année (congé de maternité ou de maladie) qui semble être un obstacle à la collaboration et à la gestion de l'organisation de l'intégration.

\section{Pistes de solutions amenées par les intervenantes du milieu}

À travers les entretiens, certaines pistes de solution ont été mentionnées. Il est possible de distinguer trois types de pistes de solutions soulevées relatives : 1) aux responsabilités de chacune; 2) à la planification structurée; 3) aux suivis concernant les modalités d'intégration de l'élève. Tout d'abord, étant donné la présence de l'éducatrice spécialisée auprès de l'élève dans les deux classes, les participantes ont soulevé l'importance de son rôle et de la nécessité de clarifier ses responsabilités dès le début de l'année scolaire concernant le soutien attendu en lien avec l'intégration. Ensuite, l'enseignante de la classe ordinaire a suggéré de prévoir une rencontre avec l'éducatrice spécialisée à chaque début de semaine afin de planifier les tâches qui seront réalisées dans la semaine et pour assurer que l'éducatrice puisse mieux soutenir l'élève. L'éducatrice a aussi mentionné cette volonté de structurer l'horaire de rencontres pour la planification de la semaine :

J'aimerais bien sûr parler de l'horaire de la semaine, évaluer le besoin de [nom de l'élève] à ce que je sois présente dans la classe, aux difficultés qui pourraient être rencontrées, trouver des stratégies ensemble pour améliorer les apprentissages de [nom de l'élève], ce qu'il est acceptable de modifier ou non. [Éducatrice spécialisée]

La directrice a reconnu que le PIA était le document commun utilisé par toutes les intervenantes et qu'il contribue à l'établissement d'objectifs communs. Toutefois, selon elle, les besoins de l'élève quant à son intégration mériteraient d'être précisés davantage et l'information devrait se retrouver dans un document de travail commun. Celle-ci explique :

Il faut se trouver du temps de qualité pour se parler de ces élèves-là, en équipe multi. Et qu'on fasse chaque fois l'évaluation, au bout d'un certain temps de ce qui se passe, de l'évolution de [ceux-ci] pour valider la pertinence de maintenir ou non les objectifs qu'on s'est donnés. [Directrice]

Cette participante a aussi ajouté des pistes d'améliorations relatives au protocole d'intégration proposé par la commission scolaire. Elle a expliqué que les échanges concernant l'intégration se déroulaient de façon informelle et elle a indiqué qu'elle souhaitait que le protocole soit davantage utilisé par les intervenants dans son école. Selon elle, la conservation des traces de façon plus formelle permettrait un meilleur arrimage des actions : "Si on veut être capable de faire un suivi, si on veut être capable d'objectiver nos décisions, il faut qu'on ait une trace de l'évolution [Directrice]. » 


\section{Adaptations du protocole d'intégration vers la classe ordinaire}

Les pistes de solutions proposées par les participantes concernaient les responsabilités respectives des intervenantes, la planification des rencontres ainsi que les suivis structurés concernant l'intégration de l'élève. Les pistes de solutions sont présentées comme des adaptations nécessaires à mettre en place en fonction des informations recueillies auprès des intervenantes du milieu scolaire étudié. Les adaptations du protocole sont donc fondées sur les besoins des intervenantes impliquées dans le processus d'intégration vers la classe ordinaire d'un élève ayant un TSA. Ces adaptations sont suggérées en vue d'atteindre le plus haut niveau d'interdépendance de Beaumont et al. (2010), celui du partage et du chevauchement d'expertise. Telles que présentées sur le tableau 2, les pistes de solutions apportées par les intervenantes interrogées ont été mises en correspondance avec les types d'échanges attendus au niveau 4 d'interdépendance du modèle théorique présenté (tableau 1).

\section{Tableau 2}

Adaptations nécessaires pour atteindre le plus haut niveau de collaboration

\begin{tabular}{lll}
\hline Niveau 4 & Types d'échanges attendus & Adaptations nécessaires \\
\hline Partage et chevauchement & Cadre commun de référence & $\begin{array}{l}\text { Lier et faire concorder le PIA et le } \\
\text { protocole d'intégration }\end{array}$ \\
\cline { 2 - 3 } & $\begin{array}{l}\text { Partage d'expertise, de ressources et } \\
\text { de responsabilités }\end{array}$ & $\begin{array}{l}\text { Établir un calendrier et les rôles de } \\
\text { chacune }\end{array}$ \\
\cline { 2 - 3 } & Résolutions de problèmes complexes & Laisser des traces des décisions prises \\
\end{tabular}

\section{Cadre commun de référence}

Les intervenantes ont mentionné qu'elles souhaitaient avoir un cadre commun de référence qui leur permettrait d'assurer la connaissance et la compréhension des objectifs poursuivis par l'intégration et ainsi assurer un arrimage cohérent entre leurs actions. Pour ce faire, une annexe dans laquelle les objectifs et besoins spécifiques de l'élève sont inscrits a été ajoutée au protocole, et ce, dès le début de l'intégration de l'élève vers la classe ordinaire. Par ailleurs, pour répondre à un besoin exprimé par les intervenantes, le nombre de rencontres préalables à l'intégration avec les parents et la direction prévues au protocole initial a été revu à la baisse, certaines rencontres ont été jumelées aux rencontres déjà prévues du PIA et sont maintenant planifiées de façon plus flexible (par exemple : des suivis par courriel).

\section{Partage d'expertise, de ressources et de responsabilités}

Au moment de la collecte, dans cette école, les rôles des intervenantes nétaient pas clairement définis, particulièrement concernant les responsabilités qui relevaient de l'éducatrice spécialisée. L'équipe a mentionné l'importance de définir, dans un contexte d'intégration vers la classe ordinaire, les attentes de chacune des intervenantes. De plus, elles ont souligné la portée de la présence de la directrice d'école pour diriger une telle discussion. Par ailleurs, pour assurer le partage de responsabilités, les intervenantes ont mentionné à plusieurs reprises la difficulté à libérer du temps pour se concerter. 
Pour pallier cet obstacle, et pour permettre des rencontres minimalement mensuelles, un calendrier des rencontres a été annexé au protocole. Les rencontres préalablement planifiées permettent aux intervenantes d'assurer une constance dans les échanges. De plus, ces suivis rigoureux permettent d'assurer de façon régulière une vérification concernant les objectifs poursuivis parl'intégration. Comme le propose Goupil (2004) alors qu'elle aborde les stratégies utiles à mettre en place lors de rencontres de PIA, en fonction des disponibilités de chacune, les intervenantes sont invitées, dès la fin de leur rencontre mensuelle, à définir la date de la rencontre subséquente, afin que toutes les intervenantes confirment leur présence et y assistent.

\section{Résolution de problèmes complexes}

Pour faciliter la résolution de problèmes complexes et pour permettre une vision à plus long terme, les intervenantes ont mentionné l'importance de conserver des traces des décisions prises tout au long du processus d'intégration de l'élève vers la classe ordinaire. Ces traces permettent d'assurer la cohérence avec les objectifs fixés et le réajustement de ceux-ci au besoin. Pour permettre ces réajustements, la période de transition où l'intégration est amorcée a été prolongée sur une période de quatre semaines pour permettre aux intervenantes d'observer comment se déroule l'intégration. Malgré la modification au protocole concernant cette phase, les intervenantes peuvent de toute évidence se rencontrer si des problématiques surviennent.

Considérant les besoins des intervenantes participantes et les adaptations nécessaires en vue de rehausser leur niveau de collaboration, des adaptations ont été apportées au Protocole d'intégration partielle vers la classe ordinaire pour un élève en classe spécialisée $e^{2}$. Il s'agit d'une démarche en trois étapes à entreprendre par la direction et l'équipe d'intervenants. Les trois étapes sont : la planification, la réalisation et le réajustement. Quatre formulaires et documents d'informations sont annexés au protocole dans sa version adaptée. Les formulaires sont utiles à la conservation des traces pour le dossier de l'élève et permettent d'assurer un suivi planifié et structuré.

\section{Discussion}

Le modèle théorique de Beaumont et al. (2010) propose un continuum basé sur quatre niveaux d'interdépendance pouvant être vécu au sein de la collaboration dans les milieux scolaires. Le niveau 4, le niveau le plus élevé, implique entre autres la planification àlong terme d'objectifs communs et la résolution de problèmes complexes. C'est dans une visée d'atteindre ce plus haut niveau d'interdépendance que des adaptations ont été suggérées pour bonifier le précédent Protocole d'intégration partielle vers la classe ordinaire pour un élève en classe spécialisée. Il semble primordial de mentionner que les participantes ont indiqué comprendre l'importance des échanges au niveau 4, le partage et le chevauchement d'expertise, mais ont mentionné que les structures organisationnelles contextuelles rendaient difficile l'atteinte de ce niveau. Cela pourrait d'ailleurs expliquer pourquoi la majorité des échanges atteignent des niveaux d'interdépendance intermédiaires (les niveaux 2 et 3). Ainsi, il est possible de croire que les mesures soutenant la collaboration proposée dans le protocole dans sa version adaptée favoriseront l'atteinte d'un plus haut niveau de collaboration. 
Le manque de temps a été mentionné à plusieurs reprises lors des entretiens avec les intervenantes. Cela confirme des résultats d'autres recherches qui rapportent que la barrière en lien avec la collaboration la plus souvent nommée est celle du temps (Caron et McLaughlin, 2002; Mitra, 2010). La problématique soulevée concernant l'attribution des responsabilités de chacune des intervenantes concorde avec les résultats de la recherche de Henry (2011). Les résultats de celle-ci ont révélé que la majorité des intervenants de l'école ne percevaient pas de répartition de tâches et affirmaient que la responsabilité de l'intégration leur revenait. Par ailleurs, certaines participantes à la recherche de Henry (2011) ont expliqué que la répartition des tâches variait d'une année à l'autre, en fonction des divers intervenants en place. Les résultats de notre recherche montrent aussi que les responsabilités de l'éducatrice spécialisée se révèlent d'une grande importance, mais que la répartition des tâches dans l'équipe n'est pas précisément établie.

La prise de notes pour assurer les suivis de l'intégration a aussi été signalée à maintes reprises. En effet, les traces écrites permettent d'assurer le suivi notamment dans le cas où l'élève vivrait un changement d'école en cours d'année ou encore une transition vers le secondaire pour l'année subséquente. Il s'avère aussi pertinent de conserver des traces des interventions déployées au sein de l'intégration dans l'éventualité où des intervenantes seraient substituées en cours d'année. La conservation des traces écrites représente une stratégie d'importance pour mieux comprendre le parcours de l'élève et les buts fixés par l'équipe. La conservation d'un tel suivi dans le dossier de l'élève permet aussi la justification des choix entrepris par l'équipe, notamment dans l'éventualité où l'intégration devrait être réajustée ou interrompue, s'il ne s'agit plus de la mesure la plus adéquate pour l'élève.

Les résultats présentés pour cette recherche-développement concernent l'adaptation d'un protocole visant progressivement une intégration totale vers la classe ordinaire d'un élève ayant un TSA, en planifiant tout d'abord une intégration partielle. L'équipe-école a toutefois relevé la pertinence de l'utilisation du protocole dans le cadre d'une intégration totale vers la classe ordinaire. Même si des ajustements concernant les modalités relatives à l'intégration totale pourraient être nécessaires, nous considérons que certaines étapes de la démarche seraient pertinentes à mettre en place dans le cadre d'une intégration de ce type.

Nous souhaitons souligner un élément à considérer en lien avec les résultats obtenus dans cette recherche-développement, notamment concernant le premier objectif soit de décrire les pratiques collaboratives lors de l'intégration partielle. Le niveau de connaissances des intervenantes interrogées relatives au trouble du spectre de l'autisme pourrait influencer leurs perceptions ainsi que leurs pratiques. En ce sens, il s'avère pertinent d'offrir du soutien aux enseignants qui intègrent des élèves ayant des besoins particuliers dans leur classe ordinaire. Du temps pour se concerter, des pratiques collaboratives encadrées ou encore de la formation axée sur les interventions appropriées auprès de ce type d'élèves sont des exemples de soutien à offrir auprès de ces enseignants (Mitra, 2010; OdierGuedj et Chatenoud, 2014). Ce soutien s'avère d'autant plus important pour les enseignants en début de carrière qui nont jamais enseigné à des élèves ayant un trouble spécifique, tel que le TSA. 
Adaptation d'un protocole de collaboration basé sur les niveaux d'interdépendance et les besoins

\section{Retombées}

Comme suggéré par Loiselle et Harvey (2007), les trois étapes de la recherche-développement (conception, réalisation et évaluation) devront faire l'objet d'une analyse plus approfondie. Les résultats présentés dans cet article relèvent des deux premières étapes de la recherche-développement, soit la conception et la réalisation. En vue d'effectuer la troisième étape, l'étape d'évaluation du protocole incluant les adaptations qui ont été développées dans le cadre de cette recherche-développement, une recherche est en cours dans cette même commission scolaire. Un des objectifs de celle-ci est de mettre à l'essai le protocole, dans sa version adaptée, dans de nouveaux milieux scolaires. Comme le mentionne Loiselle et Harvey (2007, p. 42), l'étape de l'évaluation de la recherche-développement est souvent associée à la validation du produit, mais cette étape peut aussi être utilisée pour analyser les interactions entre l'objet en cours de développement et les personnes auxquelles s'adressent cet objet. Toujours selon ces auteurs, cette perspective évaluative progressive, plutôt qu'en termes de finalité, permet l'amélioration de l'outil développé et permet d'enrichir l'analyse de "l'expérience de développement » de l'objet. Dans le cas de la recherche en cours, le protocole est mis à l'essai tout en considérant les adaptations requises propres à chaque milieu. Nous souhaitons rappeler que le protocole qui a été adapté inclut des recommandations pour une école primaire où un projet pilote concernant l'intégration vers la classe ordinaire d'élèves ayant un TSA était en cours. Les adaptations qui ont été apportées au protocole, bien qu'elles pourraient être transférables à d'autres milieux, ont été établies en fonction des besoins de cette équipe-école. D'autres milieux pourraient s'inspirer de la démarche proposée et ajuster le protocole en fonction des besoins et des ressources recueillis dans leur milieu. Malgré le fait que les résultats ne soient pas généralisables, ils pourraient soutenir d'autres milieux dans leurs réflexions en vue de mettre en place un protocole soutenant la collaboration au sein de l'intégration d'élèves ayant des besoins particuliers vers la classe ordinaire.

\section{Notes}

1 Les intervenantes qui ont pris part à cette étude de cas sont toutes des femmes. Les propos concernant nos participantes prennent donc la forme féminine.

2 Le protocole est disponible en ligne sur le site du Groupe de recherche ADEL dans la rubrique intitulée Documents à télécharger: http://www.adel.uqam.ca/outils pedagogiques.

\section{Références}

Beaumont, C., Lavoie, J. et Couture, C. (2010). Les pratiques collaboratives en milieu scolaire : cadre de référence pour soutenir la formation. Québec, QC : Centre de recherche et d'intervention sur la réussite scolaire (CRIRES), Université Laval.

Boutin, G. (1997). L'entretien de recherche qualitatif. Sainte-Foy, QC : Presses de l'Université du Québec.

Boutin, G. (2011). L'entretien de recherche qualitatif. Sainte-Foy, QC : Presses de l'Université du Québec.

Boutin, G. et Bessette, L. (2009). Inclusion ou illusion? Élèves en difficulté en classe ordinaire : Défis, limites et modalités. Montréal, QC : Éditions Nouvelles.

Caron, E. A. et McLaughlin, M. J. (2002). Indicators of beacons of excellence schools: What do they tell us about collaborative practices? Journal of Educational and Psychological Consultation, 13(4), 285-313. http://dx.doi.org/10.4324/9780203764619-2 
Gadbois, M.-È. (2015). Adaptation d'un protocole pour favoriser la collaboration dans le cas d'une intégration partielle d'un élève ayant un trouble du spectre de l'autisme à l'école primaire (Rapport d'activités et projet d'intervention). Repéré à http:// www.adel.uqam.ca/sites/default/files/rapport\%20d\%27intervention GADBOIS ME.pdf

Goupil, G. (2004). Plans d’intervention, de services et de transition. Montréal, QC : Gaëtan Morin.

Goupil, G. (2014). Les élèves en difficulté d'adaptation et d'apprentissage (4éd.). Montréal, QC : Gaëtan Morin.

Henry, M.-P. (2011). Les formes d'organisation du travail chez les enseignants du primaire qui intègrent des élèves handicapés ou en difficulté d'adaptation et d'apprentissage dans leur classe régulière (Mémoire de maitrise, Université du Québec à Rimouski). Repéré à http://semaphore.uqar.ca/id/eprint/668/

Little, J.W. (1990). The persistence of privacy: Autonomy and initiative in teachers' professional relations. Teachers college record, 91(4), 509-536.

Loiselle, J. et Harvey, S. (2007). La recherche développement en éducation : fondements, apports et limites. Recherches qualitatives, 27(1), 40-59. Repéré à http://www.recherche-qualitative.qc.ca/documents/files/revue/edition reguliere/ numero27(1)/loiselle.pdf

Miles, M. B. et Huberman, A. M. (1994). Qualitative data analysis: An expanded sourcebook (2éd.). Thousand Oaks, CA : Sage.

Ministère de l'Éducation du Québec (MEQ). (1999). Une école adaptée à tous ses élèves : politique de l'adaptation scolaire. Repéré à http://www.education.gouv.qc.ca/fileadmin/site web/documents/dpse/adaptation serv compl/politi00F 2.pdf

Ministère de l'Éducation du Loisir et du Sport (MELS). (2007). L'organisation des services éducatifs aux élèves à risque et aux élèves handicapés ou en difficulté d'adaptation ou d'apprentissage (EHDAA). Repéré à http://www.education.gouv.qc.ca/ fileadmin/site web/documents/dpse/adaptation serv compl/19-7065.pdf

Ministère de l'Éducation du Loisir et du Sport (MELS). (2010). Rencontres des partenaires en éducation. Document à l'appui de la réflexion. Rencontre sur l'intégration des élèves handicapés ou en difficulté. Québec: Gouvernement du Québec.

Mitra, S. (2010). Beliefs and practices of elementary school principals regarding collaboration between general and special education staff. (Thèse de doctorat inédite). Northern Illinois University.

Odier-Guedj, D. et Chatenoud, C. (2014). Les parents et la scolarisation d'un enfant ayant un TSA. Dans C. Chatenoud, J.-C. Kalubi et A. Paquet (dir.), La famille et la personne ayant un trouble du spectre de l'autisme. Comprendre, soutenir et agir autrement (p. 159-175). Montréal, QC : Éditions Nouvelles.

Van der Maren, J.-M. (2003). La recherche scientifique et les recherches en éducation. Dans J.-M. Van der Maren (dir.), La recherche appliquée en pédagogie (p. 15-38). Bruxelles : De Boeck.

\section{Pour citer cet article}

Gadbois, M.-E. et Dubé, F. (2020). Adaptation d'un protocole de collaboration basé sur les niveaux d'interdépendance et les besoins des intervenants au sein d'une école primaire soutenant la scolarisation d'élèves ayant un TSA en classe ordinaire. Formation et profession, 28(1), 57-68. http://dx.doi.org/10.18162/fp.2020.519 